\title{
Pancreatic neuroendocrine tumor with metastasis to the spleen: a case report
}

Yasunaru Sakuma', Yoshikazu Yasuda', Naohiro Sata', Yoshinori Hosoya', Atsushi Shimizu', Hirofumi Fujii², Daisuke Matsubara ${ }^{3}$, Noriyuki Fukushima ${ }^{3}$, Atsushi Miki ${ }^{1}$, Misato Maeno ${ }^{1}$ and Alan Kawarai Lefor ${ }^{1 *}$

\begin{abstract}
Background: Long-term term survival in patients with pancreatic neuroendocrine tumors has been reported, even in patients with metastatic disease. Metastases to the spleen are extremely rare, but have been reported from a number of primary malignancies, such as breast cancer, lung cancer, melanoma and ovarian cancer. This is the first report of a splenic metastasis from a primary pancreatic neuroendocrine tumor.

Case presentation: The patient presented as a 53 years old white male with anemia and fatigue. Physical examination revealed a left upper quadrant fullness and computed tomography showed a $24 \mathrm{~cm}$ left upper quadrant mass with multiple liver metastases, splenomegaly and a $1 \mathrm{~cm}$ mass in the spleen. Resection of the primary pancreatic tumor (T4NOM1) was accompanied by gastrectomy, splenectomy and resection of adherent bowel. The spleen contained a metastatic lesion $1.0 \mathrm{~cm}$ in diameter, consistent with a primary neuroendocrine tumor of the pancreas. This operation was followed 8 months later, by delayed resection of liver metastases. The patient receives monthly administration of somatostatin long-acting analogue and has undergone several ablations of liver lesions with percutaneous radiofrequency ablation as well as a second liver resection. The patient is alive seven years after initial presentation, with no evidence of disease on imaging studies.
\end{abstract}

Conclusions: This is the first report of a splenic metastasis from a primary pancreatic neuroendocrine tumor. The patient initially presented with synchronous multiple liver metastases and a single splenic metastasis. After resection of the primary tumor and spleen, the patient has undergone aggressive cytoreductive surgery/ ablation of liver lesions and somatostatin therapy with resulting long-term survival.

Keywords: Pancreatic neuroendocrine tumor, Spleen metastasis, Liver metastasis, Somatostatin, Radiofrequency ablation, Case report

\section{Background}

Neuroendocrine tumors occur throughout the body, and have a variety of presentations and clinical characteristics. Pancreatic neuroendocrine tumors (pNETs) are particularly rare, representing only $1-2 \%$ of all pancreatic neoplasms, but the treatment of these lesions continues to evolve with improving results. NETs are classified separately from carcinoid tumors, in part based on location [1-3]. The clinical presentation of NETs primarily depends on whether they are functional or non-functional, and if they are functional, depending on what hormone is

\footnotetext{
* Correspondence: alefor@jichi.ac.jp

${ }^{1}$ Department of Surgery, Jichi Medical University, Tochigi, Japan

Full list of author information is available at the end of the article
}

being produced [2]. Compared with NETs from other origins, patients with NETs of gastrointestinal origin are more likely to have metastases at the time of presentation [4]. The prognosis of patients with these tumors depends on a number of factors, but liver metastases are very common, being present at the time of presentation in up to $65 \%$ of patients, and are often associated with a poorer prognosis [2]. Biologic factors related to the tumor (Ki-67 index and mitotic index) have also been shown to be important and are incorporated into the $2010 \mathrm{WHO}$ grading scheme for these lesions $[2,3,5]$. Prolonged survival, even in the presence of liver metastases has been reported with aggressive treatment $[1,4]$. 
Metastases to the spleen have been reported from a variety of primary tumors. They remain extremely rare, no matter what the primary lesion is. In a meta-analysis of 713 patients with splenic metastases, Lam reported $23 \%$ from breast cancer primaries, 20\% from lung, 9\% from colon/rectum, 9\% from ovary and the rest from the stomach, prostate, and other organs [6]. The reported incidence may be increasing due to improvements in imaging technology, but they are usually found in patients with multiple visceral metastatic sites in the terminal stage [7]. Solitary splenic metastases are very unusual. The pathogenesis of metastases to the spleen is usually considered to be of arterial origin, but may be from tumor thrombi in the splenic vein [8]. The reasons for the rarity of splenic metastases rare are not understood. Mechanical factors may limit implantation of cells (angle of the splenic artery and contraction of the organ). There are also micro-environmental factors (absence of afferent lymphatics and local anti-tumor activity) which inhibit the growth of metastatic cells $[6,7]$.

We report the case of a patient who initially presented with a pNET with synchronous liver metastases and a single metastasis to the spleen. This is the first report of a metastasis to the spleen from a pNET.

\section{Case presentation}

The patient presented as a 53 years old white male with anemia and fatigue. Previous history included no medications, distal left lower extremity venous thrombophlebitis 2 months prior, status post laparoscopic gastric Roux-en-Y bypass (for obesity and diabetes mellitus which had been untreated and resolved postoperatively) three years prior to presentation and a left ulnar nerve decompression nine years prior. Physical examination revealed no palpable lymphadenopathy, a fullness in the left upper quadrant and fecal occult blood positive stool. Laboratory studies showed a serum hemoglobin of $6 \mathrm{mg} / \mathrm{dl}$. Computed tomography (CT) scan of the abdomen showed a $24 \mathrm{~cm}$ left upper quadrant mass with multiple liver lesions, splenomegaly and a $1 \mathrm{~cm}$ mass in the spleen (Fig. 1). A laparoscopic liver biopsy was performed and histopathology was initially reported as hepatocellular carcinoma versus hepatoid carcinoma. One month later, serum hemoglobin was $4.4 \mathrm{mg} / \mathrm{dl}$ and gross melena was seen. Blood transfusions did not resolve the anemia. Serum albumin was decreased to $1.7 \mathrm{~g} / \mathrm{dl}$.

The patient underwent gastrectomy, splenectomy, distal pancreatectomy and resection of small bowel and a portion of colon that were adherent to the tumor mass. At the end of surgery, there was no gross tumor remaining except for the previously identified liver metastases. Pathology showed a tumor that originated in the pancreas with direct extension to the small bowel, colon, and stomach. The resected specimen weighed

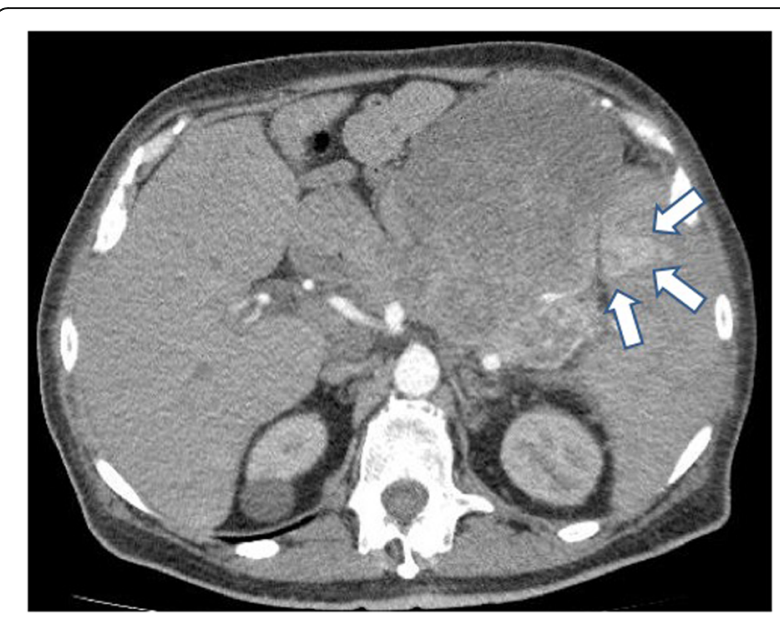

Fig. 1 Preoperative computed tomography scan of the abdomen shows a $24 \mathrm{~cm}$ mass in the left upper quadrant between the spleen and liver, and a single metastatic lesion $(1 \mathrm{~cm})$ in the spleen (arrows)

$3500 \mathrm{~g}$ and the spleen measured $14 \times 7 \times 10 \mathrm{~cm}$ (Fig. 2). On cut section, the spleen contained a $1.0 \mathrm{~cm}$ lesion consistent with a metastasis, which was then confirmed to be a metastatic lesion on histopathologic evaluation (Fig. 3).

The final pathology report showed a pNET, well differentiated, stage T4NOM1 with 0/13 lymph nodes containing tumor. The lesion in the spleen was located near the edge of the spleen, but there was no direct invasion by the tumor mass (Figs. 2 and 3) grossly or histologically. Examination of the splenic vein showed no evidence of thrombosis with only minimal invasion by the tumor histologically. There were three mitoses per 50 high power fields, and Ki- $67<1 \%$. Perineural and lymphovascular invasion was seen. Immunostains for chromogranin A

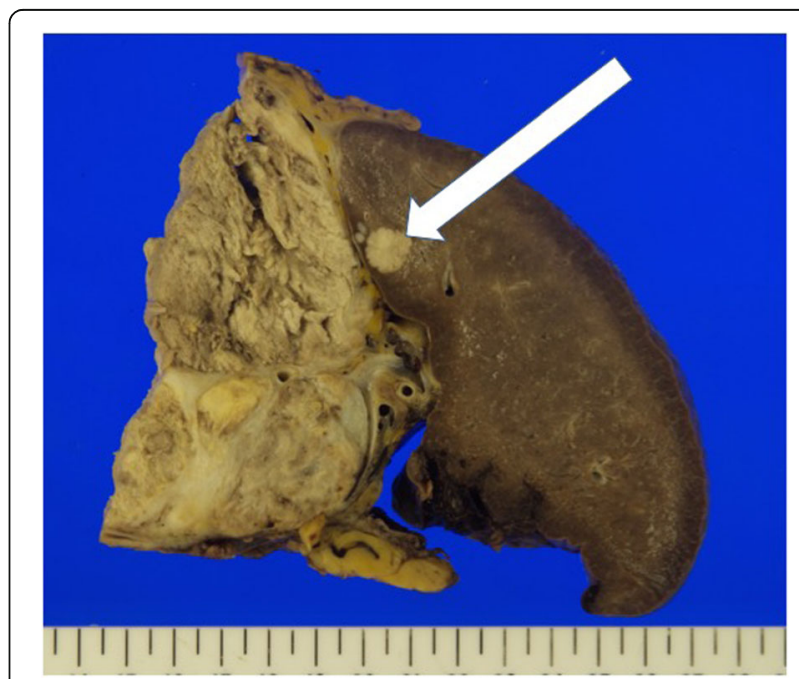

Fig. 2 Gross pathology image of the cut surface of the spleen and tumor showing a $1.0 \mathrm{~cm}$ metastasis (arrow), corresponding to the lesion seen on the computed tomography scan 


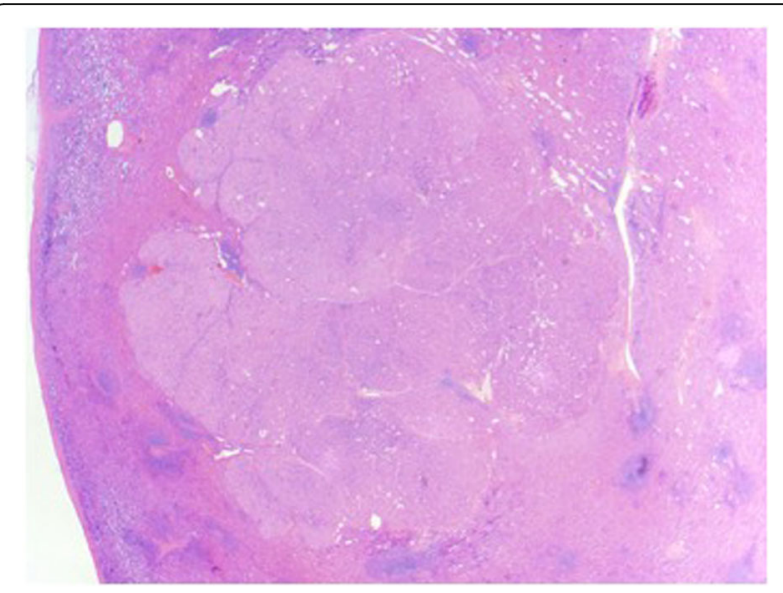

Fig. 3 Histopathology of the spleen lesion, with an appearance similar to the histopathology of the primary lesion in the pancreas. The metastatic lesion extends close to, but not through the splenic capsule and is separate from the adjacent tumor. (Hematoxylin and eosin stain, 15X)

and synaptophysin are positive (Fig. 4). Immunostains for CD56, OCGH1E5, AFP, glucagon, insulin, somatostatin, pancreatic polypeptide, alpha 1-anti trypsin, alpha 1 antichymotrypsin and lipase are negative. Blood chemistry studies showed that this was a non-functional tumor.

The patient was discharged home ten days after surgery and returned two weeks later with intra-abdominal sepsis treated with drainage of fluid and antibiotics. Diabetes mellitus recurred in this patient after pancreatic resection. Somatostatin administration, long-acting release $20 \mathrm{mg} / \mathrm{month}$ was started. Approximately 8 months after the resection of the primary lesion, repeat imaging studies (CT scan, magnetic resonance imaging) and an octreotide nucleotide scan showed no lesions outside the liver. Left lateral segmentectomy was performed with simultaneous resection of multiple hepatic nodules throughout the liver. All lesions were consistent with metastases from the previously resected primary tumor on histopathologic examination.

The patient was discharged without complications, and continued monthly somatostatin therapy as well as periodic CT scans to evaluate recurrence. About one year after liver resection, a new lesion appeared in the liver which was treated with percutaneous radiofrequency ablation (RFA). The patient did well until 14 months later when multiple liver lesions appeared. A second liver resection was completed, including resection of nine nodules $(<1 \mathrm{~cm}$ size $)$ and RFA of two central liver lesions. It was felt that all lesions visible were treated at that time. Seventeen months later, another liver lesion appeared which was centrally located and treated with percutaneous RFA. An octreotide scan showed no evidence of disease. About 20 months later another central area in the liver was treated with percutaneous RFA.

The patient is now seven-years status post resection of the primary lesion, treated with monthly administration of somatostatin long acting release $20 \mathrm{mg} \mathrm{IM}$, and followed with periodic CT scans of the liver. Diabetes mellitus persists and is treated with insulin. At seven years, there is no evidence of residual disease in the abdomen, or within the parenchyma of the liver. He remains asymptomatic and is doing well. The patient's clinical history is summarized in the Table 1, prepared in accordance with CARE guidelines [9].

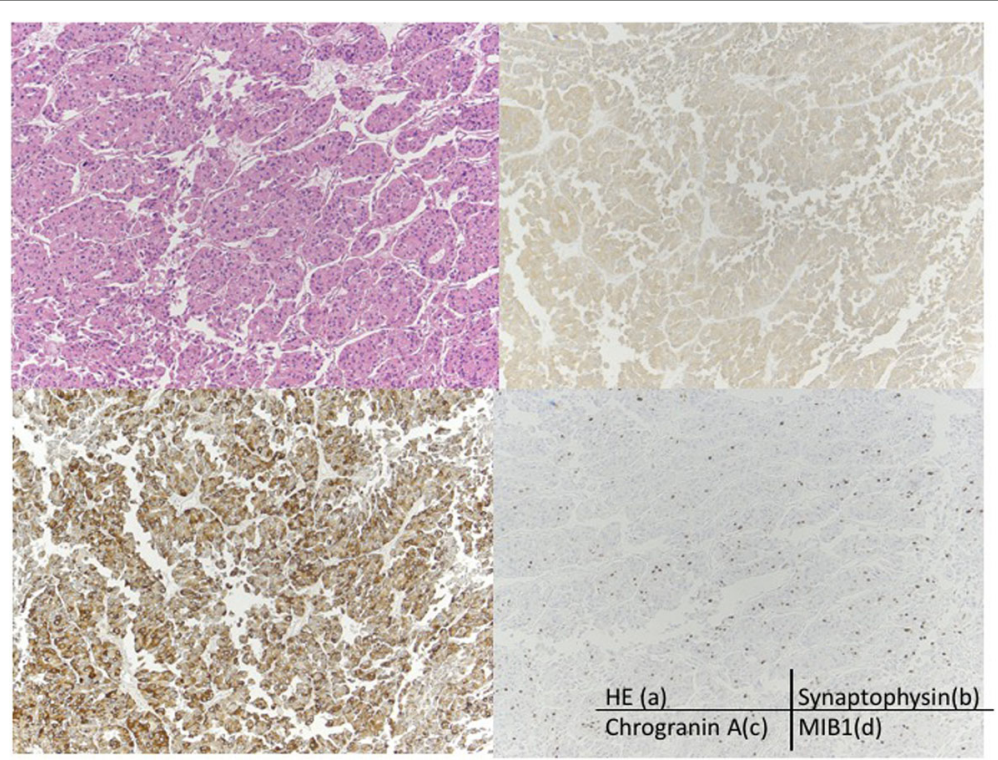

Fig. 4 a Histopathological findings revealed that the tumor was composed of cells with round nuclei arranged in sheets or rosettes (Hematoxylin and eosin stain $\times 100)$. b The tumor cells were weakly positive for synaptophysin by immunohistochemical staining $(\times 100)$, c The chromogranin A ( $\times 100)$, and MIB 1 index was about 10\% (d) In this case, CD56 and other immunohistochemical stains were negative except for SSTR-2 (x100) 
Table 1 Timeline of care

\begin{tabular}{|c|}
\hline rior History \\
\hline $\begin{array}{l}-9 \text { years: Left ulnar nerve decompression } \\
\text { - }-3 \text { years: Roux-en-Y gastric bypass for obesity with resolution of previously } \\
\text { untreated Type } 2 \text { Diabetes Mellitus }\end{array}$ \\
\hline Diagnosis and Interventions \\
\hline $\begin{array}{l}\text { - Anemia, fatigue, left upper quadrant fullness on physical examination, } \\
\text { serum hemoglobin } 7 \mathrm{mg} / \mathrm{dl} \\
\text { - CT scan showed a } 24 \mathrm{~cm} \text { left upper quadrant mass with multiple liver } \\
\text { metastases and a single } 1 \mathrm{~cm} \text { spleen metastasis } \\
\text { - Laparoscopic liver biopsy: read as hepatocellular carcinoma vs. } \\
\text { hepatoid carcinoma } \\
\text { - +1 month: Serum hemoglobin } 4.0 \mathrm{~g} / \mathrm{dl} \text {, surgery for completion } \\
\text { gastrectomy, splenectomy, small bowel resection, colon resection, and } \\
\text { distal pancreatectomy, complicated by intra-abdominal sepsis treated } \\
\text { non-operatively }\end{array}$ \\
\hline Follow-up and outcomes \\
\hline $\begin{array}{l}-+8 \text { months: left lateral segmentectomy of the liver, resection of } \\
\text { multiple liver nodules } \\
\cdot+20 \text { months: solitary liver lesion treated with percutaneous RFA } \\
\cdot+35 \text { months: multiple liver lesions, open resection with intraoperative } \\
\text { RFA } \\
\cdot+52 \text { months: Percutaneous RFA of central liver lesion } \\
\cdot+77 \text { months: Negative octreotide scan, percutaneous RFA of central } \\
\text { liver lesion } \\
\cdot+84 \text { months: No evidence of disease, monthly administration of long } \\
\text { acting release somatostatin ( } 20 \mathrm{mg} \text { ) and diabetes mellitus (treated with } \\
\text { insulin) }\end{array}$ \\
\hline
\end{tabular}

CT Computed tomography, RFA radiofrequency ablation

\section{Discussion}

Neuroendocrine tumors are rare, but their reported incidence has increased over the last decades, which is attributed to improved diagnostic modalities and surveillance $[4,7]$. In one study, the incidence from 1974 to 2004 more than doubled, from 1.4 new cases per million to 3.0 [10]. Gastrointestinal neuroendocrine tumors have a higher likelihood of presenting with metastases, which are present in about $50-65 \%$ of patients at the time of diagnosis. With the availability of new treatment modalities, long-term survival is reported, even in patients who present with metastatic disease. A recent review showed that the combination of long-acting somatostatin analogue therapy with aggressive cytoreductive surgery can result in long-term survival in some patients with metastatic NETs originating in the gastrointestinal tract [4].

In a series of 46 patients with pNETs, $48 \%$ presented with loco-regional disease and $52 \%$ presented with metastases [11]. All patients without metastases underwent resection, and the authors report a three-year survival of $86 \%$ with a median 42 months follow-up. Patients with metastatic disease were treated with a variety of modalities including resection, ablation, chemoembolization and others, and had a three-year survival of $70 \%$. The authors credit their use of a multidisciplinary multimodal approach. An aggressive approach to these lesions was also reported by Touzios et al [1], who categorized the treatment of 60 patients with pNET into three groups: no treatment, resection, and transarterial chemoembolization. Five-year survival was $25 \%$ in the no treatment group, $72 \%$ for the resection group and 50\% for the transarterial treated group. They also found poor outcomes in patients with $>50 \%$ involvement of the liver.

The impact of lymph node involvement on survival in patients with pNET was studied by Krampitz and colleagues [12]. They found that disease-related survival decreased as a function of the number of lymph nodes involved. However, in patients with liver metastases and lymph node metastases, the major determinant of survival is the presence of liver metastases. The probability of survival at 10 years is $30 \%$ in this series. In a large series of patients with non-functional pNETs, tumor size and nodal status were not associated with survival, while tumor grade and systemic metastases were associated [10].

Somatostatin receptor scintigraphy is an important modality in the evaluation of these patients, and peptide receptor radionuclide therapy has shown promising results in the therapy of these lesions [13]. Transplantation has been used in select patients with results similar to transplantation performed for hepatocellular carcinoma [14]. These authors advise waiting for stabilization of liver disease prior to proceeding to transplantation. However, transplantation is generally not considered as a first-line therapy for metastatic neuroendocrine tumors [4].

The presence of splenic metastases is usually reported in the context of a pre-terminal event with multiple visceral metastases. In this patient, it is of interest that the single spleen metastasis was found at the initial presentation. The origin of the splenic metastasis is the subject of some speculation. In the spleen, it is believed that reticulo-endothelial create an unfavorable environment for the growth and survival of tumor cells $[6,7,15]$. Splenic metastases are believed to originate from the splenic artery, splenic vein, or lymphatics [8]. However, there are few lymphatic ducts to the spleen, and most metastases are considered of hematogenous origin [16]. Marymont et al demonstrated that splenic metastases are seen in the venous sinusoids and /or red pulp, supporting a hematogenous origin [17]. The splenic artery would appear to be the most common origin of splenic metastases although this suggests systemic circulation of tumor cells, and metastases in other organs may be expected, making solitary splenic metastases less likely. In patients with pNET, liver metastases from the portal vein are extremely common. In the present patient, although histologic evaluation of the portal vein showed no evidence of portal vein thrombus, the huge tumor mass and minimal invasion of the splenic vein seen on histologic evaluation, may have affected portal vein outflow, leading to congestion of the splenic vein and possibly resulting in development of a splenic metastasis [18]. 
In patients with late appearance of splenic lesions, treatment may be futile but splenectomy may benefit patients who experience significant pain due to splenomegaly. Isolated splenic metastases were extremely rare in a series of 92 splenic metastases, representing just $4 \%$ of the lesions [6]. In that series, $26 \%$ of the spleens weighed more than $200 \mathrm{~g}$. It is estimated that only about 100 cases of isolated splenic metastases have been reported [19], from diverse primary tumors. Splenectomy for isolated metastases can result in long term survival [7]. It has been suggested that splenic metastases may result from the growth of early blood-borne tumor cells, after a latent period [7]. Laparoscopic splenectomy is a reasonable surgical option in a patient with an isolated splenic metastasis.

Despite an extensive review of the literature including multiple databases (keywords "spleen", "metastases", "neuroendocrine"), there are no reports of patients with metastases to the spleen from pNETs. In one review of unusual locations for metastases from neuroendocrine tumors, there were no lesions metastatic to the spleen [20]. Metastases to the spleen have been reported for bronchial carcinoid tumors [21, 22]. The spleen has been reported to be involved with neuroendocrine tumors of the pancreas, including a tumor thrombus in the splenic vein without a mass in the spleen [23] and as a splenic mass from direct extension and gastric varices [24]. The rarity of splenic metastases is not entirely surprising, since pNETs and splenic metastases are both rare entities.

\section{Conclusions}

This is the first report of a splenic metastasis from a primary pancreatic neuroendocrine tumor. There are a number of interesting features in the present patient's clinical history. The spleen metastasis in this patient was solitary and found at initial presentation, rather than as a pre-terminal event. This patient had no lymph node metastases at initial presentation which is unusual in the presence of liver metastases. This patient has undergone aggressive cytoreductive treatment and monthly administration of long-acting somatostatin analogue that have likely contributed to the continued survival of this patient.

\section{Abbreviations}

CT: Computed tomography; NET: Neuroendocrine tumor; pNET: Pancreatic neuroendocrine tumor; RFA: Radiofrequency ablation

\section{Acknowledgements}

The patient requested this opportunity to acknowledge the excellent care provided by many physicians not listed as co-authors, who contributed to his long-term survival. This paper was prepared in accordance with the CARE guidelines [9].

\section{Funding}

There was no funding for this paper.

\section{Availability of data and materials}

All data in this paper is confidential patient information which is kept as part of the official medical record at the site of care.

\section{Authors' contributions}

All authors read and approved the final manuscript. Review of patient data and critical comments were performed by $\mathrm{YY}, \mathrm{NS}, \mathrm{YH}, \mathrm{HF}, \mathrm{AS}$ and $\mathrm{AM}$. The literature review was completed by YS, MM, and AL. DM and NF reviewed and described the pathologic findings. The manuscript was written by YS, $\mathrm{AS}, \mathrm{MM}$ and $\mathrm{AL}$.

\section{Competing interests}

The authors declare that they have no competing interests.

\section{Consent for publication}

Written consent for publication was obtained from the patient described and is available for review.

Ethics approval and consent to participate

Not applicable as this is not a study. The need for approval was waived by the Jichi Medical University Institutional Review Board.

\section{Author details}

${ }^{1}$ Department of Surgery, Jichi Medical University, Tochigi, Japan. ${ }^{2}$ Department of Oncology, Jichi Medical University, Tochigi, Japan. ${ }^{3}$ Department of Pathology, Jichi Medical University, Tochigi, Japan.

Received: 6 November 2016 Accepted: 16 December 2016

Published online: 09 January 2017

\section{References}

1. Touzios JG, Kiely JM, Pitt SC, Rilling WS, Quebbemann EJ, Wilson SD, Pitt HA. Neuroendocrine hepatic metastases: Does aggressive management improve survival? Ann Surg. 2005;241:776-85.

2. Metz DC, Jensen RT. Gastrointestinal neuroendocrine tumors: Pancreatic endocrine tumors. Gastroeneterol. 2008:135:1469-92.

3. Klimstra DS, Modlin IR, Coppola D, Lloyd RV, Suster S. The pathologic classification of neuroendocrine tumors: A review of nomenclature, grading and staging systems. Pancreas. 2010;39:707-12.

4. Deutsch GB, Lee JH, Bilchick AJ. Long term survival with long acting somatostatin analogues plus aggressive cytoreductive surgery in patients with metastatic neuroendocrine carcinoma. J Am Coll Surg. 2015;221:26-36.

5. Dhall D, Mertens R, Bresee C, Parakh R, Wang HL, Li M, et al. Ki-67 proliferative index predicts progression-free survival of patients with welldifferentiated ileal neuroendocrine tumors. Human Pathol. 2012;43:489-95.

6. Lam KY. Metastatic tumors to the spleen: A 25 year clinicopathologic study. Arch Pathol Lab Med. 2000;124:526-30.

7. Comperat E, Bardier-Dupas A, Camparo P, Capron F, Charlotte F. Splenic metastases: Clinicopathologic presentation, differential diagnosis and pathogenesis. Arch Pathol Lab Med. 2007;131:965-9.

8. Berge T. Splenic metastases Frequencies and patterns. Acta Pathol Microbiol Scand. 1974;82:499-506

9. Gagnier JJ, Kienle G, Altman DG, Mosher D, Sox H, Riley D, the CARE Group. The CARE guidelines: consensus-based clinical case report guideline development. J Clin Epidemiol. 2014;67:46-51.

10. Franko J, Feng W, Yip L, Genovese E, Moser AJ. Non-functional neuroendocrine carcinoma of the pancreas: Incidence, tumor biology, and outcomes in 2158 patients. J Gastrointest Surg. 2010;14:541-8.

11. Nissen NN, Kim AS, Yu R, Wolin EW, Friedman ML, Lo SK, Wachsmann AM, Colquhoun SD. Pancreatic neuroendocrine tumors: Presentation, management and outcomes. Am Surgeon. 2009;75:1025-9.

12. Krampitz GW, Norton JA, Poultsides GA, Visser BC, Sun L, Jensen RT. Lymph nodes and survival in pancreatic neuroendocrine tumors (pNET). Arch Surg. 2012;147:820-7.

13. Teunissen JJM, Kwekkeboom DJ, Valkema R, Krenning EP. Nuclear medicine techniques for the imaging and treatment of neuroendocrine tumors. Endocr-relate Cancer. 2011;18:S27-51.

14. Gedaly R, Daily MF, Davenport D, McHugh PP, Koch A, Angelo P, Hundley JC. Liver transplantation for the treatment of liver metastases from neuroendocrine tumors. Arch Surg. 2011;146:943-8.

15. Warren S, Davis AH. Studies on tumor metastasis. The metastasis of carcinoma to the spleen. Am J Cancer. 1934;21:517-33.

16. Abi Saad GS, Hussein M, El-Saghir NS, et al. Isolated splenic metastasis from colorectal cancer. Int J Clin Oncol. 2011;16:306-13. 
17. Marymont $\mathrm{Jr} \mathrm{JH}$, Gross S. Patterns of metastatic cancer in the spleen. Am J Clin Pathol. 1963;40:58-66.

18. Koga H, Takami Y, Toyosaki R, et al. One case of metachronous non-solitary splenic metastasis from colon cancer. Rin to Ken. 2006;83:423-6.

19. Arif A, Abideen ZU, Zia N, Khan MA, Nawaz T, Malik AZ. Metastatic involvement of the spleen by endometrial adenocarcinoma; a rare asylum for a common malignancy: a case report. BMC Res Notes. 2013;6:476-7.

20. Naswa N, Sharma P, Kumar R, Malhotra A, Bal C. Usual and unusual neuroendocrine tumor metastases on 68Ga-DOTANOC PET/CT. Clin Nuc Med. 2013;38:e239-45.

21. Balmforth D, Skouras C, Palazzo F, Zacharakis E. Laparoscopic management for carcinoid metastases to the spleen. HPB Surg. 2011;346507.

22. Carreras C, Kulkarni HR, Baum RP. Rare metastases detected by 68Gasomatostatin receptor PET/CT in patients with neuroendocrine tumors. Recent Results Cancer Res. 2013:194:379-84.

23. Rodriguez $\mathrm{R}$, Overton $\mathrm{H}$, Morris $\mathrm{KT}$. Pancreatic neuroendocrine tumor with splenic vein tumor thrombus: A case report. Int I Surg Case Rep. 2014;5:1271-4.

24. Shah SA, Amarapurkar AD, Prabhu SR, Kumar V, Gangurde GK, Joshi R. Splenic mass and isolated gastric varices: A rare presentation of a neuroendocrine tumor of the pancreas. J Pancreas. 2010;11:444-5.

Submit your next manuscript to BioMed Central and we will help you at every step:

- We accept pre-submission inquiries

- Our selector tool helps you to find the most relevant journal

- We provide round the clock customer support

- Convenient online submission

- Thorough peer review

- Inclusion in PubMed and all major indexing services

- Maximum visibility for your research

Submit your manuscript at www.biomedcentral.com/submit
Biomed Central 